Study of the spatial di stribution of the Langmii $r$ wave ampl itude in the turbul ent pl asma usi ng el ectron beam shadowgr aph techni que

\begin{tabular}{|c|c|}
\hline 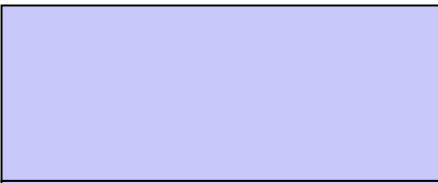 & $\begin{array}{l}\text { Ando Ri toku, Fuj i i H sashi, Wada Sat oshi, } \\
\text { Toyosugi Nor i o, Nor i shi t a H r omasa, Okanmt o } \\
\text { Naoki, Nasuzaki Nasar u, Kamada Kei i chi }\end{array}$ \\
\hline 著者別表示 & 安藤 利得, 増崎 克, 鎌田 啓一 \\
\hline $\begin{array}{l}\text { j our nal or } \\
\text { publ i cat i on } \mathrm{title}\end{array}$ & $\begin{array}{l}\text { BEANS } 2002 \text { - 14t h I nt er nat i onal Conf er ence on } \\
\text { H gh- Power Part i cl e Beans }\end{array}$ \\
\hline vol une & 650 \\
\hline page $r$ ange & 381- 384 \\
\hline year & 2002-01- 01 \\
\hline URL & ht t p: //hdl . handl e. net /2297/32469 \\
\hline
\end{tabular}




\title{
Study of the Spatial Distribution of the Langmuir Wave Amplitude in the Turbulent Plasma using Electron Beam Shadowgraph Technique
}

\author{
Ritoku Ando, Hisashi Fujii, Satoshi Wada, Norio Toyosugi, Hiromasa Morishita, \\ Naoki Okamoto, Masaru Masuzaki, and Keiichi Kamada
}

Faculty of Science, Kanazawa University, 920-1192, JAPAN

\begin{abstract}
New diagnostic, “electron-beam-shadowgraph”, is examined for taking the spatial distribution of transient fields in a plasma. It is applied to the unmagnetized plasma, which is perturbed by an intense relativistic electron beam (IREB). Strong deflection is observed with duration of about 1 $\mu \mathrm{s}$ just after the IREB injection. The shadowgraph image is characterized by presence of aggregate holes. The size of the hole is changed with the plasma density, and corresponds to the 15 - 60 Debye lengths. Important agreements are observed between the experiment and the strong Langmuir turbulence theory.
\end{abstract}

\section{INTRODUCTION}

An electron beam excites electric field by the instabilities when it passes through a plasma. If the beam is dense enough, the power density of the electric field becomes also high comparable to the thermal energy of the plasma electron. The oscillating field with the near plasma frequency is unstable, then localized spatially and becomes bumpy. The state is called the strong Langmuir turbulence, in which many collapsing nonlinear wave packets, called the "caviton"s are produced.

Normal electron-beam probing technique employs a beam with a small cross-section. The local electric field in the plasma is estimated from the deflection angle of the beam. It is difficult to obtain the spatial distribution of the transient electric field. New diagnostic, "electron-beam-shadowgraph", with a large cross-section is examined. The shadowgraph of the unmagnetized plasma, which is perturbed by an intense relativistic electron beam (IREB), is taken.

\section{EXPERIMENTAL SETUP}

We have developed an electron beam probing technique applicable to IREB-plasma system. The "shadowgraph" technique is a kind of the beam probing with employing a beam with a large cross-section. An improvement of the detector which increases the signal-to-noise ratio, $\mathrm{S} / \mathrm{N}$, makes it possible to use the lower current density beam. The outline of the electron probing system used for this experiment is shown in Fig.1. An electron gun for the probing was set at the position $z=305 \mathrm{~mm}$, where $z$ is the distance from the anode. The probing beam propagates perpendicularly to the main chamber. An electron-beam has current of $\sim 30 \mathrm{~mA}$, energy of $50 \mathrm{kV}$, and pulse length of $100 \mathrm{~ns}$. Because the probing beam is defocused, the 
beam diverges and the cross-section increases with the distance from the gun. The distance from the electron gun to the center of the main chamber is $220 \mathrm{~mm}$, and that from the center to the phosphor screen is $346 \mathrm{~mm}$. The projection magnifies the object at the center 2.6 times. The orifices are set along the path of the probing beam as shown in Fig.1 to reduce the IREB influx to the screen and the electron gun. The diameter of the probing beam is about $70 \mathrm{~mm}$, which is limited by the first orifice placed at the

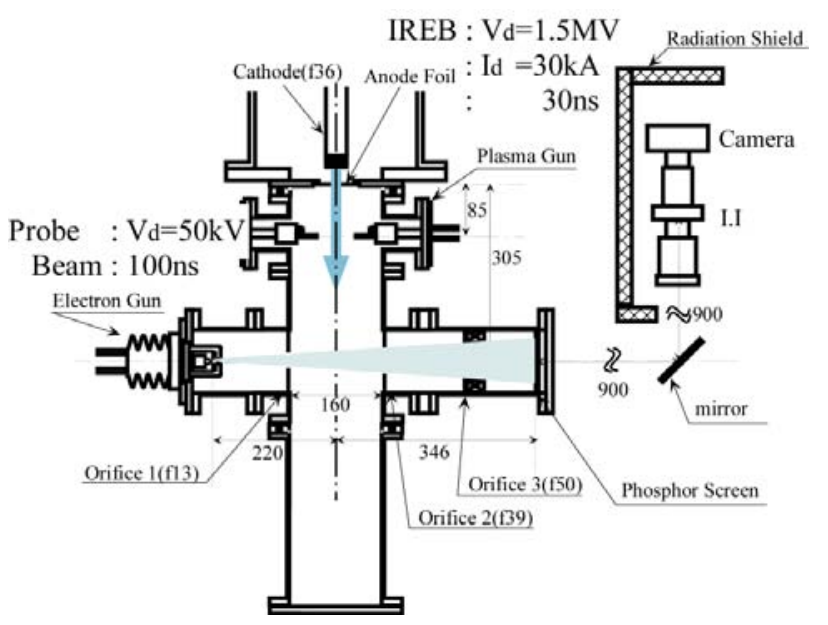

FIGURE 1. Experimental setup entrance of the main chamber. The phosphor screen is set larger than the beam diameter. In this experiment, the time resolution for the measurement is set longer than the Langmuir oscillation period but shorter than the timescale of the ion movement. In this setting, the probing beam is deflected by both static and oscillating electric fields, which deflect and spread out the beam on principle.

An IREB with energy of $1.5 \mathrm{MeV}$, current of $30 \mathrm{kA}$, and duration of $30 \mathrm{~ns}$ is injected to the plasma. A drift chamber, $600 \mathrm{~mm}$ long and $160 \mathrm{~mm}$ in diameter, is used. No external magnetic field is applied. The rail-type plasma guns with carbon rods are set at the position of $z=100 \mathrm{~mm}$. The plasma is produced by the rail-type guns and its after-glow plasma is utilized. The plasma density, $n_{\mathrm{p}}$, was measured with a microwave interferometer and Langmuir probes. The $n_{\mathrm{p}}$ is a function of the time, $\tau$, from a start of the discharge. The $n_{\mathrm{p}}$ at $z$ $=175 \mathrm{~mm}$ reaches $3 \times 10^{13} \mathrm{~cm}^{-3}$ at $\tau=12 \mu \mathrm{s}$. After the plasma reaches at the chamber wall at $\tau=20 \mu \mathrm{s}$, the $n_{\mathrm{p}}$ is distributed with a gentle slope in the chamber and decrease exponentially in time. The main data presented in this paper is obtained with the timing of $\tau=50 \mu \mathrm{s}, 60 \mu \mathrm{s}$, $80 \mu \mathrm{s}, 100 \mu \mathrm{s}$, with plasma density of $n_{\mathrm{p}}=4 \times 10^{11} \mathrm{~cm}^{-3}, 1 \times 10^{11} \mathrm{~cm}^{-3}, 4 \times 10^{10} \mathrm{~cm}^{-3}$ and $2 \times 10^{10}$ $\mathrm{cm}^{-3}$ respectively. The main component of the plasma is probably carbon, but it contains other components, like hydrogen or oxygen as the impurities.

\section{EXPERIMENTAL RESULTS}

The examples of the beam pattern are shown in Fig.3. The dark part on the picture is the location where the electrons hit the screen. Fig.3(a) shows the beam pattern without deflection in the case of a vacuum. The cross-section of the beam is

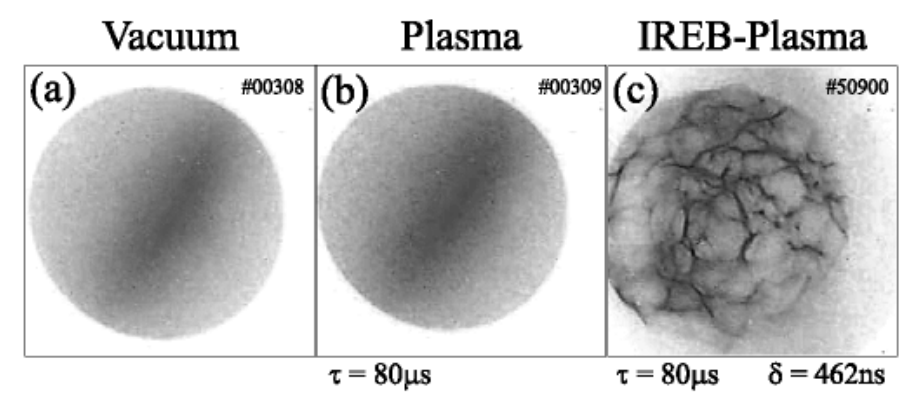

FIGURE 2. Examples of the beam pattern before and after the deflection 
observed as a large dark circle in the pictures. The beam density is not perfectly homogeneous. The alteration of the spatial distribution of beam density from the original beam pattern is measured.

The probing beam is not affected by the presence of undisturbed plasma, as shown in Fig.2(b). But the pattern is changed strongly by the participation of the IREB injection. Many holes with various sizes are observed in Fig.2(c). The typical shadowgraph image is characterized by appearance of aggregate holes. From another point of view, the pattern behind the holes is a mesh. It is an irregular mesh. The mesh size from a few-millimeter to few-centimeters is observed through the experiment. It is observed that the mesh size, $b$, strongly depends on the $n_{\mathrm{p}}$. It becomes larger when the $n_{\mathrm{p}}$ decreases, i.e., as the increase of the $\tau$, as shown in Fig.3. The deflection is observed with duration of about $1 \mu$ s just after the IREB injection. The parameter $\delta$ is taken as the time interval from the beginning of IREB pulse to the beginning of the probing beam. Following dependency on the $\delta$ is observed. Figure 4 shows the typical change of the pattern with the time transition under a fixed $n_{\mathrm{p}}$. (1) Clear image of the hole is not observed for any shot at $\delta<400$ ns. (2) Holes are clearly recognized at $\delta=450-700$ ns. The size changes in this time duration as shown in Fig.5. (3) At $\delta=700-1600 \mathrm{~ns}$, the picture of the holes becomes thinner. The holes with small circular shape are observed. (4) It becomes impossible to observe any deflection after $\delta>1600 \mathrm{~ns}$.

The perturbation is decreased by reducing the IREB current. A masking plate with many holes is attached to the anode foil for reducing the IREB current. The beam density dependence in the case of $\tau=100 \mu$ s is shown in Fig.6. The transmittance rate is changed between $4 \%$ and $100 \%$. The $n_{\mathrm{b}} / n_{\mathrm{p}}$ is estimated about between 0.023 and 0.29 . The aggregate holes gradually disappear as the IREB current decreases. A number of holes cover the whole surface in every case when it appears. The mesh size decreases with the decrease of the $n_{\mathrm{b}}$. The perturbation is small enough to recognize on the image for $n_{\mathrm{b}} / n_{\mathrm{p}}=0.023$.

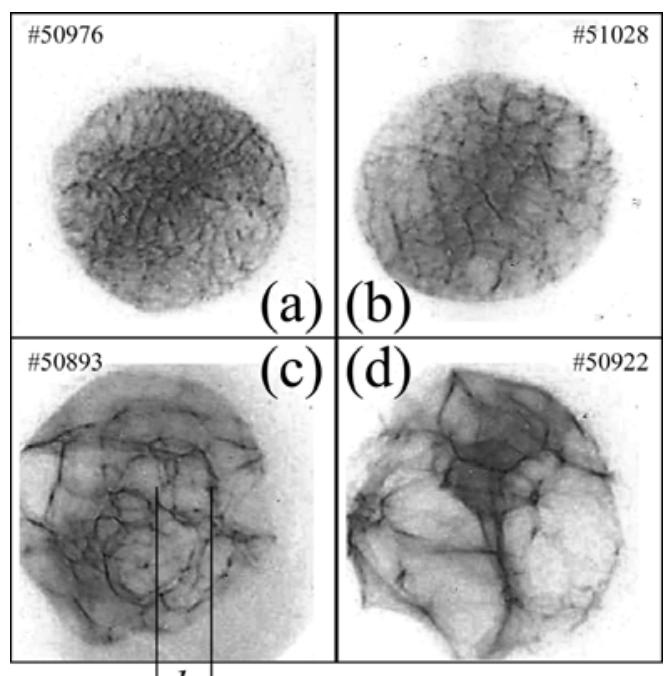

FIGURE 3. The plasma density dependence. (a) $\tau=50 \mu \mathrm{s}$, (b) $60 \mu \mathrm{s}$, (c) $80 \mu \mathrm{s}$, (d) $100 \mu \mathrm{s}$. The diameter of the hole is measured from one mesh to the next mesh, which is mentioned as the mesh size, $b$. in this paper.

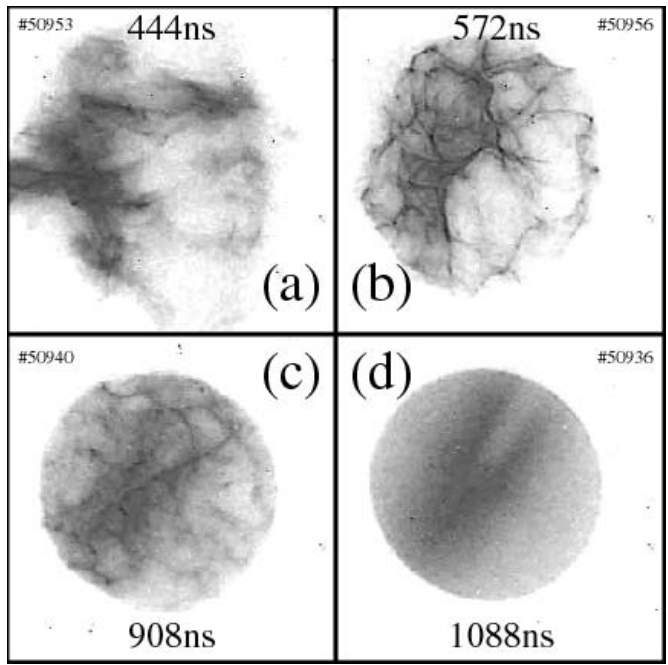

FIGURE 4. Typical shadowgraph after an IREB passed through. (a) $\delta=444 \mathrm{~ns}$, (b) 572ns, (c) 908ns, and (d) 1088ns. The plasma density is $2 \times 10^{11} \mathrm{~cm}^{-3}$, and the beam density is about $10^{10} \mathrm{~cm}^{-3}$. 


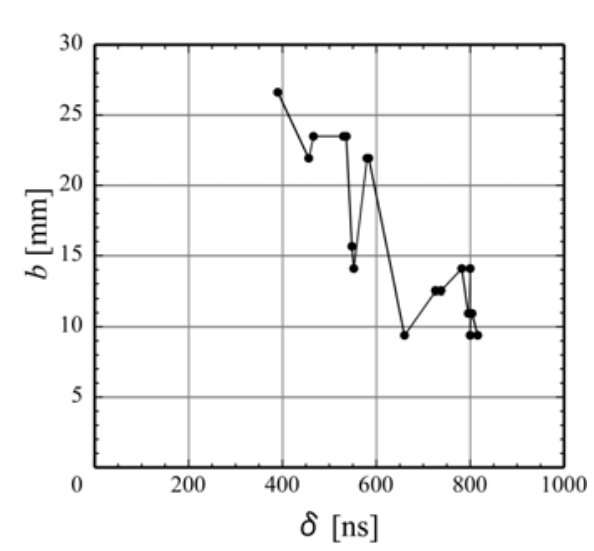

FIGURE 5. The mesh size $b$ decreases with $\delta$.

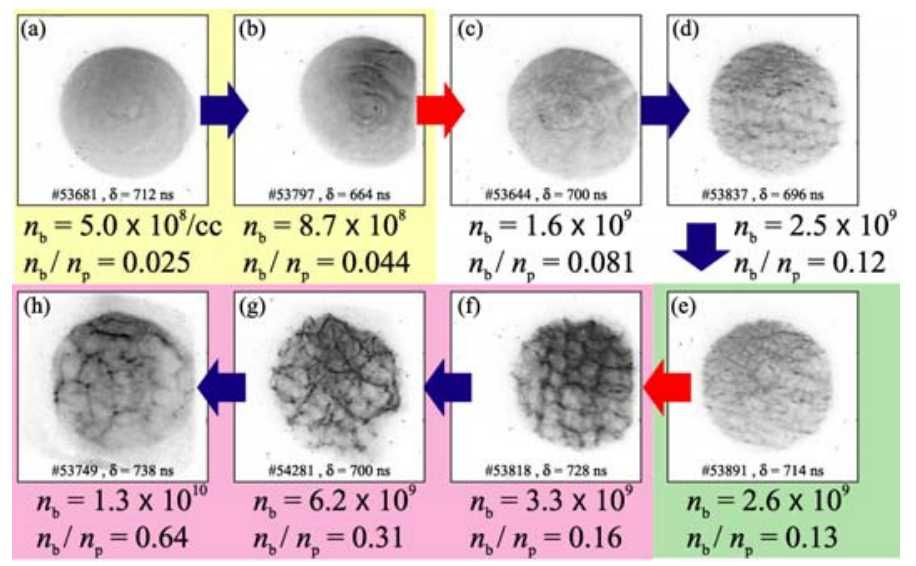

FIGURE 6. The beam density is decreased with keeping plasma density constant, $\tau=100 \mu \mathrm{s}$.

\section{CONCLUSIONS}

The electron-beam-shadowgraph image of the strongly disturbed plasma by IREB was always characterized by presence of aggregate holes. The various dependences on basic parameters were investigated experimentally. Those dependences can be explained by assuming the proper "caviton" field. A measurement of the accurate electron temperature probably justify the caviton model and improve the reliability of the diagnostic. Application of this new diagnostic to other phenomena is expected.

\section{ACKNOWLEDGEMENTS}

A part of this work is supported by a Grant-in-Aid for Scientific Research from Ministry of Education, Science, Sports and Culture, Japan.

\section{References}

1. P. A. Robinson, Rev. Modern Phys., 69, 507-573 (1997).

2. Ando, R., et. al., IEEE trans. Plasma Sci., 27, 1545-1547 (1999).

3. Ando, R., et. al., Proc. BEAMS2000, 893-896 (2000)

4. Ando, R., et. al., Research Report NIFS PROC-50, ISSN 0915-6348, 163-172 (2001). 